\title{
Preparation and Properties of Biphasic Calcium Phosphate Scaffolds Multiply Coated with HA/PLLA Nanocomposites for Bone Tissue Engineering Applications
}

\author{
Lei Nie, Jinping Suo, Peng Zou, and Shuibin Feng \\ State Key Laboratory of Material Processing and Die and Mould Technology, College of Materials Science and Engineering, \\ Huazhong University of Science \& Technology, No. 1037 Luoyu Road, Wuhan 430074, China \\ Correspondence should be addressed to Jinping Suo, jinpingsuo@mail.hust.edu.cn
}

Received 9 January 2012; Accepted 30 March 2012

Academic Editor: Tianxi Liu

Copyright $\odot 2012$ Lei Nie et al. This is an open access article distributed under the Creative Commons Attribution License, which permits unrestricted use, distribution, and reproduction in any medium, provided the original work is properly cited.

A well-developed BCP scaffolds coated with multilayer of HA/PLLA nanocomposites with interconnectivity, high porosity, and moderate compressive strength as well as good biocompatibility were fabricated for bone tissue engineering. After being multiply coated with HA/PLLA nanocomposites, the scaffolds maintained the BCP framework structure, and the porous network structure of scaffolds remained unchanged; however, the compressive strength was increased with the increase of coating layer number of HA/PLLA nanocomposites. The prepared scaffolds showed lower variation of $\mathrm{pH}$ values in SBF solution, and an increase of coating layer number led to the decrease of the biodegradation rate at different days. Moreover, the multilayer coating scaffolds had good cytocompatibility, showing no negative effects on cells growth and proliferation. Furthermore, the bone-like apatite layer was built obviously in the interface of scaffold after 21 days after implantation in SD rat muscle. In conclusion, the BCP scaffold coated with multilayer of HA/PLLA nanocomposites could be a candidate as an excellent substitute for damaged or defect bone in bone tissue engineering.

\section{Introduction}

Bone is a living tissue, which continuously rebuilds its structure. Thanks to its capacity of spontaneous regeneration, most lesions, such as fractures, heal well with conventional therapy. However, in case of large defects and osseous congenital deformities, a bone graft or a bone substitute is needed to assist healing [1]. Due to source limitation of the autogenetic bone graft and immunologic rejection problem of the allergenic bone graft [2], bone tissue engineering technique has become a crucial strategy to permanently repair bone defects. Over the past decade, considerable effort has been focused on fabricating the three-dimensionally interconnected macroporous scaffold to guide bone reconstruction through rapid vascularization, bone growth, and remodeling. In order to allow for optimal osteogenesis and the formation of new bone throughout the scaffolds and the surrounding tissue [3], 3D scaffolds need to have the appropriate physical, biological, and mechanical properties.
These include pore size, pore structure, surface topography, chemical composition, degradation, and mechanical strength. However, from the material point of view, no existing scaffold possesses excellent synergistic properties of high mechanical strength, suitable degradation rate, high porosity, and excellent bioactivity [4-6], which constitutes the main challenge to the use of traditional biomaterials in bone tissue engineering. Thus, the new scaffolding material systems as well as new fabricating techniques are the most important demands in the bone tissue engineering field.

Bioceramics have been widely studied and recognized as the most promising materials for the development of scaffolds for bone regeneration [7]. Biphasic calcium phosphate (BCP) ceramics composed of hydroxyapatite (HA) and $\beta$-tricalcium phosphate $(\beta$-TCP) have been extensively studied in the past two decades $[8,9]$. HA has excellent biocompatibility and bioactivity and can be directly bonded to the host bone [10], $\beta$-TCP has suitable degradation rate that matches the growth rate of newly formed bone [11], and 
by combination of the advantages of HA and $\beta$-TCP, BCP ceramics have been recently developed as an excellent starting material to prepare the bone tissue engineering scaffold. Moreover, BCP ceramics showed excellent osteoinductivity, osteoconductivity, bioactivity, and biodegradability [12] according to in vitro physiochemical property examinations and in vivo biological evaluations. However, the inherent brittleness of BCP ceramics makes them unsuitable in loadbearing bone applications [13]. Different methods have been applied to overcome these drawbacks. One of the effective methods for enhancing the mechanical properties and bioactivity of ceramic scaffolds is coating the struts of scaffolds with biocompatible materials, while maintaining the macropores intact and open. The coatings used so far are ceramics, polymers, and micron-composite materials [14-22]. Composites have shown to be more effective for enhancement of both mechanical properties and bioactivity, compared to ceramic and polymers. To the best of the authors' knowledge, nanocomposite coating layers on the surface of highly porous ceramic scaffolds are yet to be produced. In the present study, nanocomposite coatings consisting of poly-L-Lactide (PLLA) as a polymer matrix and nanohydroxyapatite (HA) as ceramic filler were used to coat the BCP scaffolds [23-29]. Polylactic acid is an FDAapproved biodegradable polymer with remarkable toughness and good biocompatibility. HA is the major mineral component ( $69 \%$ wt.) of human hard tissues. HA promotes faster bone regeneration and direct bonding to regenerated bones without intermediate connective tissues. The incorporation of HA in a polymeric matrix has to overcome processing and dispersion challenges since it is of a great interest to the biomedical community. Sui et al. [30] fabricated PLLA/HA composite scaffolds via electrospinning and concluded that the cell adhesion and growth on the PLLA/HA composite scaffolds were far better than those on the pure PLLA scaffolds. Jeong et al. [31] prepared the PLA/HA composite nanofibrous scaffolds and showed that MC3T3-E1 cells maintained viability and proliferated continuously for up to 21 days, suggesting that the PLA/HA composites are effective scaffolds for the growth of osteoblasts. Roohani-Esfahani et al. [32, 33] prepared the biphasic calcium phosphate scaffold coated with HA-PCL composites and found that gaps between inorganic particles and polymer matrix could provide a channel for the penetration of water into polymer led to the $\mathrm{pH}$ change in SBF solution and weight loss of scaffolds; however, the mechanical strength of scaffolds was unsatisfactory for load-bearing bone. In this study, we fabricated highly porous BCP scaffolds using a polymeric reticulate method and multiply coated them with HA/PLLA nanocomposites to improve the mechanical strength. The developed scaffolds were characterized for their mechanical properties and biological degradation and for the efficacy of multilayer coatings in enhancing the bioactivity of the scaffolds.

\section{Experimental}

2.1. Materials. PVA was obtained from the Shanxi Sanwei Group Co. Ltd. (polymerization $\sim 1799$ and degree of hydrolysis $\sim 99 \%)$. Diammonium hydrogen phosphate $\left(\left(\mathrm{NH}_{4}\right)_{2} \mathrm{HPO}_{4}\right)$ and calcium nitrate tetrahydrate $\left(\mathrm{Ca}\left(\mathrm{NO}_{3}\right)_{2} \cdot 4 \mathrm{H}_{2} \mathrm{O}\right)$ were obtained from the Sinopharm Chemical Reagent Co. Ltd. Ammonia was obtained from the Wuhan Chemical Reagent Co. Ltd. All the chemical reagents used in this work were in analytical reagent level.

\subsection{Fabrication of BCP Nanoparticles and BCP Porous} Scaffold. Calcium phosphate-deficient apatite powder was prepared by aqueous precipitation reaction. Briefly, $\mathrm{Ca}\left(\mathrm{NO}_{3}\right)_{2} \cdot 4 \mathrm{H}_{2} \mathrm{O} \quad(0.4 \mathrm{M})$ and $\left(\mathrm{NH}_{4}\right)_{2} \mathrm{HPO}_{4} \quad(0.2 \mathrm{M})$ solutions were added in a three-necked flask by simultaneous drops at room temperature while the $\mathrm{pH}$ was adjusted at 11 using buffers; the resultant white color product was washed several times with distilled water to completely remove the buffer. The powder obtained was thermally treated at $1100^{\circ} \mathrm{C}$ for $1 \mathrm{~h}$, to produce a biphasic calcium phosphate that consisted of about $40 \% \mathrm{HA}$ and $60 \% \quad \beta$-TCP. The BCP powder was crushed in a mortar and pestle and classified using stainless steel sieves to provide particles of size $<75 \mu \mathrm{m}$.

Fully reticulated polyurethane foam was used as a sacrificial template for the replication method. For preparing the slurry, PVA was dissolved in water, the concentration being $0.1 \mathrm{~mol} / \mathrm{L}$. Then BCP powder was added to PVA-water solution up to concentration of $30 \mathrm{wt} \%$. PU foam templates were cut to appropriate dimensions, and the surface layer of the sponge was treated in $\mathrm{NaOH}$ solution for $30 \mathrm{~min}$ to improve the surface layer's hydrophilicity. After cleaning and drying, the PU sponges were immersed in the BCP slurry and compressed slightly to facilitate its absorption. Excessive slurry was removed by squeezing, and the remaining slurry was subsequently blown with compressed air to obtain a uniform dispersion on the sponge. After drying at $37^{\circ} \mathrm{C}$ for $48 \mathrm{~h}$, BCP-coated sponges were fired in air in an electric furnace using a 4-stage schedule, which include heating from $25^{\circ} \mathrm{C}$ to $600^{\circ} \mathrm{C}$ with a heating rate of $1{ }^{\circ} \mathrm{C} / \mathrm{min}$, further heating from $600^{\circ} \mathrm{C}$ to 1200 at $2^{\circ} \mathrm{C} / \mathrm{min}$, dwelling at $1200^{\circ} \mathrm{C}$ for $2 \mathrm{~h}$, and finally cooling down to $25^{\circ} \mathrm{C}$ at the cooling rate of $5^{\circ} \mathrm{C} / \mathrm{min}$.

\subsection{Multilayers of HA/PLLA Nanocomposites Coating on BCP} Porous Scaffold. As poly-L-lactide powder (PLLA; Mw = 200,000, Sigma-Aldrich) is insoluble in water, the polymer was first dissolved in 1,4-dioxane at $80^{\circ} \mathrm{C}$ for $1 \mathrm{~h}$, until complete dissolution. Afterwards, HA nanopowder and purified water were added (dioxane/water $=87 / 13$, HA/PLLA = $3 / 10$ ) and stirred for $24 \mathrm{~h}$ until a homogeneous clear solution was obtained and then sonicated for 1 min just before coating the scaffolds. The prepared BCP scaffolds were cleaned with ethanol dipped into the composite solutions for $1 \mathrm{~min}$, and then excess solution was removed to form a uniform coating. The coated scaffolds were dried for 3 days in an oven at $37^{\circ} \mathrm{C}$ followed by 1 day in a fume hood. In this way, the BCP scaffolds coated with monolayer were prepared, and the BCP scaffolds coated with multilayers were prepared by repeating the above procedure.

2.4. Physicochemical Characterization of the Prepared Scaffolds. Firstly, the BCP nanoparticles were characterized 
TABLE 1: Order, amounts, weighing container, and formula weight of reagents for preparing $1000 \mathrm{~mL}$ of SBF.

\begin{tabular}{lcccc}
\hline Order & Reagent & Amount & Container & Formula weight \\
\hline 1 & $\mathrm{NaCl}$ & $8.035 \mathrm{~g}$ & Weighing paper & 58.4430 \\
2 & $\mathrm{NaHCO}_{3}$ & $0.355 \mathrm{~g}$ & Weighing paper & 84.0068 \\
3 & $\mathrm{KCl}$ & $0.225 \mathrm{~g}$ & Weighing bottle & 74.5515 \\
4 & $\mathrm{~K}_{2} \mathrm{HPO}_{4} \cdot 3 \mathrm{H}_{2} \mathrm{O}$ & $0.231 \mathrm{~g}$ & Weighing bottle & 228.2220 \\
5 & $\mathrm{MaCl}_{2} \cdot 6 \mathrm{H}_{2} \mathrm{O}$ & $0.311 \mathrm{~g}$ & Weighing bottle & 203.3034 \\
6 & $1.0_{\mathrm{M}}-\mathrm{HCl}$ & $39 \mathrm{~mL}$ & Graduated cylinder & 110.9848 \\
7 & $\mathrm{CaCl}_{2}$ & Weighing bottle & 142.0428 \\
8 & $\mathrm{Na}_{2} \mathrm{SO}_{4}$ & $0.292 \mathrm{~g}$ & Weighing bottle & 121.1356 \\
9 & $\mathrm{Tris}$ & $0.072 \mathrm{~g}$ & Weighing paper & \\
10 & $1.0_{\mathrm{M}}-\mathrm{HCl}$ & $6.118 \mathrm{~g}$ & Syringe & \\
\hline
\end{tabular}

by FE-scanning electron microscopy (FSEM) (Sirion 200, Holland FE). The cross-section of the porous scaffolds were coated with gold to prevent imaging artifacts from electrical charging. And their morphologies were examined by FEIscanning electron microscopy (SEM) (Quanta 200, Holland FEI). The pore size was calculated from SEM pictures by selecting five arbitrary areas for measurement. Furthermore, FTIR (VERTEX, Bruker) was used to characterize the presence of specific chemical groups in BCP scaffolds coated with HA/PLLA nanocomposites. FTIR spectra were obtained within the range between 4000 and $500 \mathrm{~cm}^{-1}$ on VERTEX 70 FTIR spectrometer with a resolution of $1 \mathrm{~cm}^{-1}$, using attenuated total reflectance (ATR) technique. In addition, the prepared samples were analyzed by X-ray diffraction (X-Pert PRO, PANalytical B.V.). This instrument works with voltage and current settings of $40 \mathrm{kV}$ and $40 \mathrm{~mA}$, respectively, and uses $\mathrm{Cu}-\mathrm{K} \alpha$ radiation $(1.540600 \AA)$. For qualitative analysis, XRD diagrams were recorded in the interval $10^{\circ} \leq 2 \theta \leq$ $90^{\circ}$ at scan speed of $2^{\circ} \% \mathrm{~min}$. The open porosity can be calculated by the liquid displacement method. The scaffold was submerged in a known volume $\left(V_{1}\right)$ of ethanol that was not a solvent for the scaffold, and a series of brief evacuation repressurization cycles was conducted to force the liquid into the pores of the scaffold. After these cycles, the volume of the liquid and liquid-impregnated scaffold was $V_{2}$. When the liquid-impregnated scaffold was removed, the remaining liquid volume was $V_{3}$, and open porosity (p) was calculated as $\left(V_{1}-V_{3}\right) /\left(V_{2}-V_{3}\right)$. Besides, the compressive strength was determined by crushing a $10 \times 10 \times 10 \mathrm{~mm}^{3}$ scaffold between two flat platens using electromechanical universal testing machine (SANSCMT4503, China) with a ramp rate of $0.5 \mathrm{~mm} \mathrm{~min}^{-1}$. Five identical specimens for each sample group were used for porosity and the compressive testing.

2.5. Biodegradation in Simulated Body Fluid. The biodegradation of the prepared scaffolds in vitro was investigated by soaking them in SBF solution at $37^{\circ} \mathrm{C}$. The SBF solution was prepared according to the procedure described in Table 1 [34]. Cylinder-shaped scaffolds were immersed in SBF solution at $37^{\circ} \mathrm{C}$ for $1,5,10,15,20$, and 25 days at a solid/ liquid ratio of $50 \mathrm{mg} / \mathrm{mL}$. All samples were held in plastic flasks which were sealed to minimize the change in $\mathrm{pH}$ and microorganism contamination. The SBF solution was not refreshed during the experiment. After soaking, the samples were filtrated, rinsed with distilled water, and dried at $40^{\circ} \mathrm{C}$ for 4 days and the final weight of samples carefully measured. The weight loss was calculated according to percentage of initial weight. Five scaffolds were used to measure the weight loss and $\mathrm{pH}$ change, and results were expressed as mean \pm SD.

2.6. In Vitro Cytotoxicity Testing: MTT Assay. The immortalized rat osteoblastic ROS 17/2.8 cell line (obtained from the Orthopedics Department, Union Hospital, Tongji Medical College of Huazhong University of Science and Technology) was utilized in this study. These cells were incubated in a humidified atmosphere of $5 \% \mathrm{CO}_{2}$ at $37^{\circ} \mathrm{C}$. Cells were cultured in Dulbecco's modified Eagle's medium (DMEM, Invitrogen, Paisley, UK) and supplemented with $10 \%$ fetal bovine serum (FBS, Invitrogen, Paisley, UK), $100 \mathrm{mg} / \mathrm{mL}$ streptomycin, and $100 \mathrm{U} / \mathrm{mL}$ penicillin. The culture medium was changed every alternate day. The cytotoxicity of the prepared was investigated by the MTT (3-[4,5-dimethylthiazol2-yl]-2,5-diphenyltetrazolium bromide) assay which was used to assess cell proliferation by measuring mitochondrial succinate dehydrogenase activity. The samples were fixed in each bottom of a 24-well cell culture plate and were sterilized with ethylene oxide (ETO) steam for $24 \mathrm{~h}$ at room temperature; then $1 \mathrm{~mL}$ cell suspension was seeded evenly onto each sample. The culture medium was changed by fresh medium every 2 days. After seeding for 1, 3, and 5 days, $100 \mu \mathrm{L}$ of MTT $(5 \mathrm{mg} / \mathrm{mL})$ solution was added to each well and incubated at $37^{\circ} \mathrm{C}$ for $4 \mathrm{~h}$ to allow the formation of formazan crystals; respectively, after removing supernatants, $650 \mu \mathrm{L}$ of dimethyl sulfoxide (DMSO) was added to each well for dissolving the blue formazan crystal; then the solution was transferred to 96-well plates. The absorbance of each well was measured at $570 \mathrm{~nm}$ using an ELISA microplate reader (Bio-Rad). The cells without scaffold were served as the negative control in this study. A mean value was obtained from the measurement of four test runs. The results were statistically analyzed to express the mean 5 the standard deviation (SD) of the mean.

2.7. In Vivo Experiment: Muscle Implantation. Ten healthy Sprague-Dawley (SD) rats of $200 \mathrm{~g}$ were randomly divided 


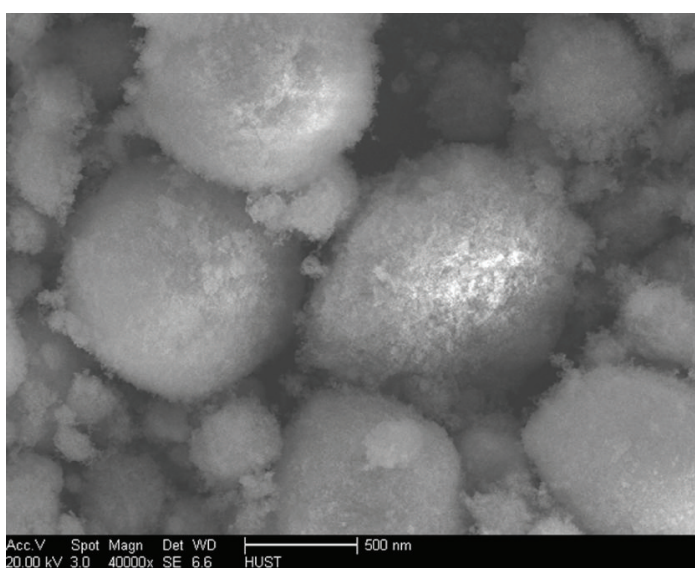

(a)

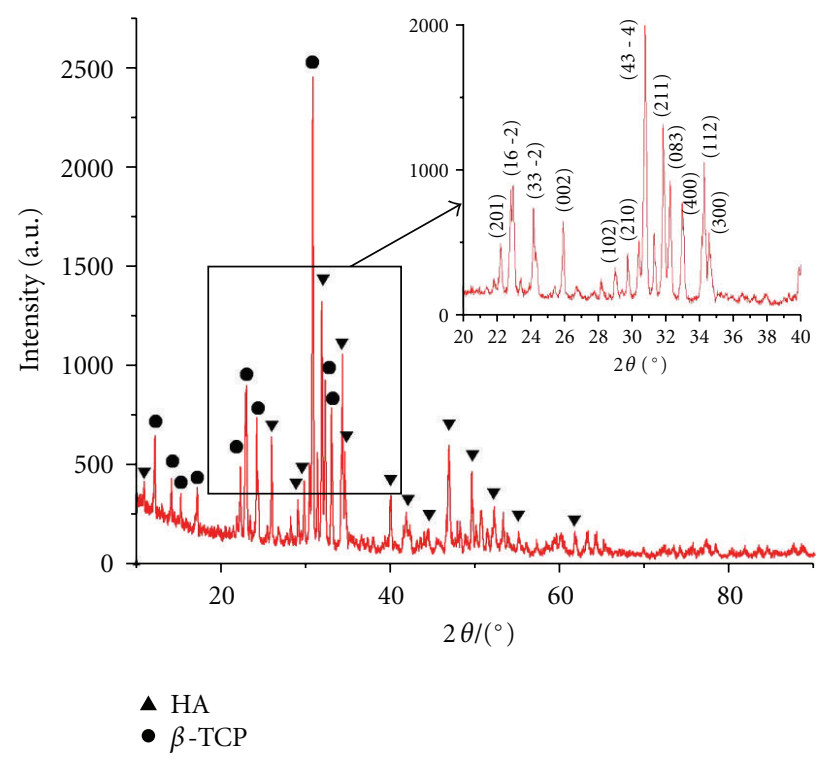

(b)

FIGURE 1: FSEM image (a) and XRD analysis (b) of BCP nanopowders.

into five groups. The rats were anaesthetized using an intraperitoneal injection of $0.3 \mathrm{wt} \%$ pentobarbital sodium. A muscle pouch about $8 \mathrm{~mm}$ in length was created along the fiber direction in the thigh muscle of the rat under surgical asepsis condition. After the implantation of onecolumn sample in the muscle pouch of each animal, the wound was closed with suture and disinfection. Penicillin was injected in the following 1 day, and the stitches were taken out in 7 days after implantation. The animals were observed to affirm whether there existed any wound injection and abnormal reaction. Two rats were executed by breaking the neck at consecutive time period of $1,7,14$, 21 , and 28 days, respectively, and the related tissues were separated and retrieved. Then samples were fixed with $10 \%$ neutral formalin, undecalcified sectioned, HE (hematoxylin and eosin) stained and analyzed histologically under optic microscope.

2.8. Statistical Analysis. All the statistical data are expressed as the mean $\pm \mathrm{SD}$ and were obtained from at least five independent experiments. For statistical analysis, first Levene's test was performed to determine the homogeneity of variance for all the data, and then, the Tamhane post hoc test was performed for the comparison between different groups. PASW statistics program was employed for all statistical analysis, and differences were the data was accepted at a $P$ value of $<0.05$ for $95 \%$ confidence.

\section{Results and Discussion}

3.1. Physical Properties of BCP Nanopowders. The BCP nanopowders properties were investigated by XRD and FSEM analysis in our study. Figure 1(a) shows the distinct high-resolution FSEM morphologies of the BCP nanoparticles prepared in this study. The BCP nanoparticles were agglomerated as spherical form, and the diameter of spherical grain was in the range of $0.5 \sim 1 \mu \mathrm{m}$. It was necessary that the stirring hours were long enough in the preparation process of BCP slurry to disperse BCP particles in PVAwater solution uniformly. Existence of nanoparticulate phase in solid ceramic matrix is known as a method of achieving enhanced mechanical strength and toughness of the matrix [35]. It was found that the BCP scaffold was crisp while the prepared BCP particle size was greater than $75 \mu \mathrm{m}$. The nanoparticles serve to steer cracks to propagate along the paths of higher toughness. The toughness at grain boundaries is lower than that within grains. The internal stress produced by the nanoparticles at grain boundaries tends to alter the crack propagation path from intergranular to transgranular and thus increases the overall toughness of the material. Furthermore, nanoparticles will also improve sinterability and reduce flaw size [36]. Figure 1(b) shows the XRD pattern of BCP powders treated at $1100^{\circ} \mathrm{C}$. The HA and $\beta$-TCP peaks were present in prepared BCP samples, the peaks at $2 \theta=25.9^{\circ}(002), 28.2^{\circ}(102), 29.7^{\circ}(210), 31.8^{\circ}$ (211), 32.3 (112), and $34.6^{\circ}$ (202) matching closely with the diffraction peaks of stoichiometric HA (JCPDS no. 9-0432). The peaks of $\beta$-TCP appear at $2 \theta=22.9^{\circ}\left(\begin{array}{lll}3 & 3 & -2\end{array}\right), 24.2^{\circ}\left(\begin{array}{lll}3 & 6 & -1\end{array}\right)$, $30.8^{\circ}\left(\begin{array}{lll}4 & 3 & -4\end{array}\right), 34.2^{\circ}\left(\begin{array}{lll}3 & 3 & -5\end{array}\right)$, and $34.6^{\circ}\left(\begin{array}{lll}4 & 2 & -1\end{array}\right)$ (JCPDS no. 9-0169). XRD pattern showed that no other impurity was found in the BCP nanopowders. By quantitative analysis for the XRD patterns, it was confirmed that biphasic calcium phosphate that consisted of about $40 \%$ HA and $60 \% \beta$-TCP was acquirable. BCP is more effective in bone repair or regeneration than pure HA or pure $\beta$-TCP and has a controllable degradation rate.

3.2. Physicochemical Characterization of the Prepared Scaffolds. The XRD patterns of pure HA and HA/PLLA composite compared to BCP are shown in Figure 2. As presented 


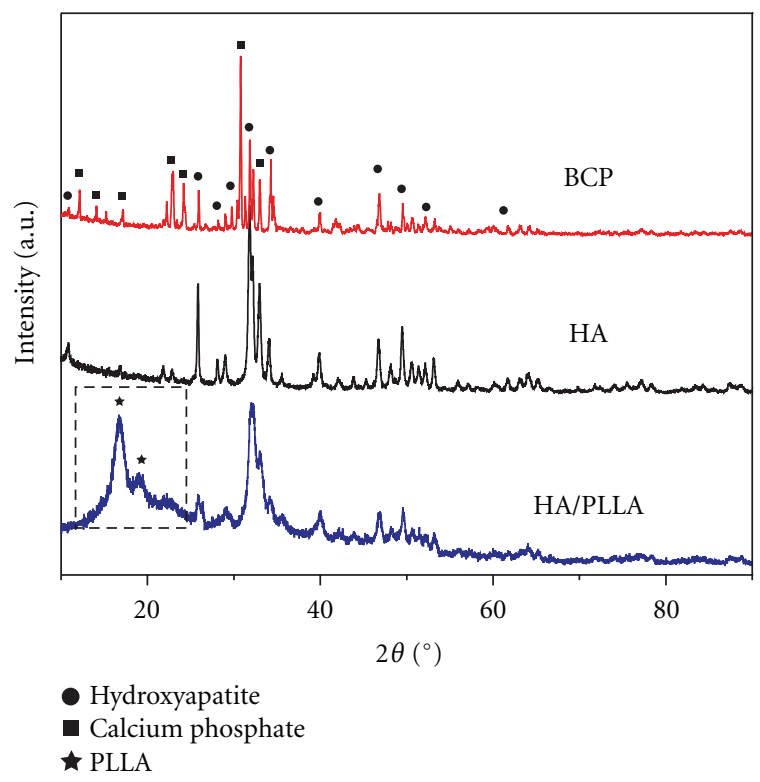

FIGURE 2: XRD patterns of BCP nanopowders, HA nanopowders, and HA/PLLA composites.

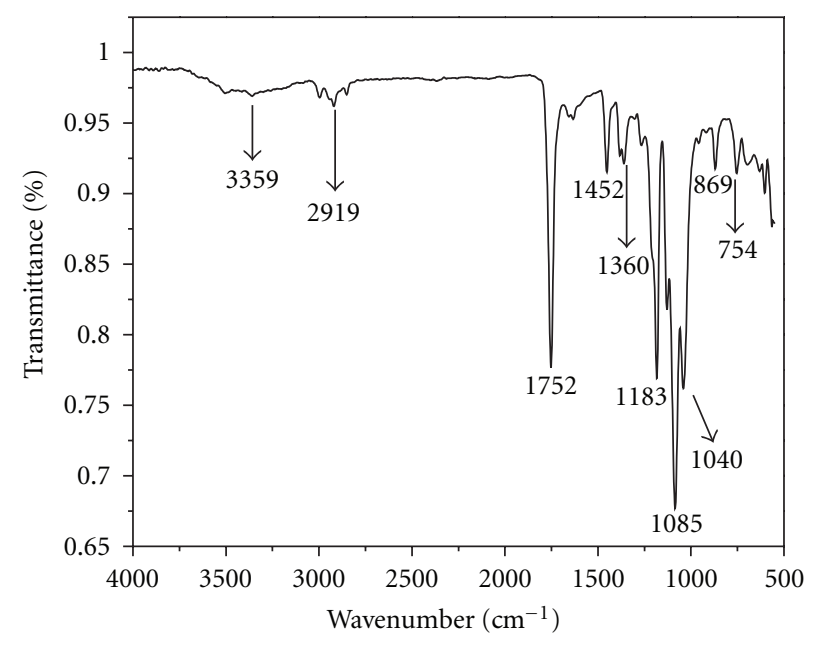

FIGURE 3: FTIR spectra of HA/PLLA nanocomposites.

in Figure 2, the synthetic HA has a poorly crystalline nature with peaks at $2 \theta=26^{\circ}(002), 28.7^{\circ}(210), 31.8^{\circ}(211)$, $32.3^{\circ}(112)$, and $34.6^{\circ}$ (202) matching closely with the diffraction peaks of stoichiometric HA, which also present in the sintered BCP sample. XRD data show the crystalline structures of PLLA, and the diffraction peaks for PLLA were at $2 \theta=17.49^{\circ}$ and $19.31^{\circ}$ [37].

The absorption spectrum of the HA/PLLA nanocomposites was shown in Figure 3. The phosphate ions, $\mathrm{PO}_{4}{ }^{3-}$, were the principal molecular components of BCP contributing to the IR absorbance in the $1200-550 \mathrm{~cm}^{-1}$ regions. The characteristic peaks at 1040 and $1085 \mathrm{~cm}^{-1}$ correspond to the stretching vibration of $\mathrm{PO}_{4}^{-3}$ and at $599 \mathrm{~cm}^{-1}$ to the deformation vibrations of $\mathrm{PO}_{4}^{-3}$. As with other major components, the characteristics of the $\mathrm{OH}^{-}$bands in $\mathrm{HA}$ were identified by observing the broad band from about 3700 to $2500 \mathrm{~cm}^{-1}$. The peak of this band was centered at about $3131 \mathrm{~cm}^{-1}$, which was a typical assignment of the stretching mode of $\mathrm{OH}^{-}$ions. In addition, the two distinct bands were observed at 3571 and $1752 \mathrm{~cm}^{-1}$ which arise from the stretching mode and bending mode of $\mathrm{H}_{2} \mathrm{O}$ molecules, respectively. Furthermore, the characteristics of carbonyl group and methyl in PLLA were identified by observing the band at 1452 and $1360 \mathrm{~cm}^{-1}$, respectively.

3.3. Scaffold Morphology and Microstructure Analysis. Figure 4 shows the macroporous network morphologies and strut microstructure of the biphasic calcium phosphate scaffolds before (a) and after coating with different multilayer of HA/PLLA nanocomposites ((b) to (f)). The expected $3 \mathrm{D}$ interconnected porous structure with open macropores was observed in the SEM pictures, and the pore sizes of open macropores were about $300-600 \mu \mathrm{m}$. The average pore size of the macropores of the scaffolds was approximately $450 \mu \mathrm{m}$. For BCP scaffold without coating (Figure 4(a)), the pore wall was coarse and the small pores were observed. After coating BCP with HA/PLLA nanocomposites, the scaffolds maintained the BCP framework structure, and the porous network structure of scaffolds remained unchanged. However, the surface morphologies of scaffolds have changed significantly. A uniform coating was found over the surface of BCP, and the crack-like defects as well as microspores in the struts were filled by nanocomposites. After coating BCP scaffold with monolayer of HA/PLLA nanocomposites (Figure 4(b)), the strut of the scaffold showed a smooth surface. As can be seen in Figure 4(c), the cracks on the scaffold surface were observed while BCP coated with two layers of HA/PLLA nanocomposites. Furthermore, 

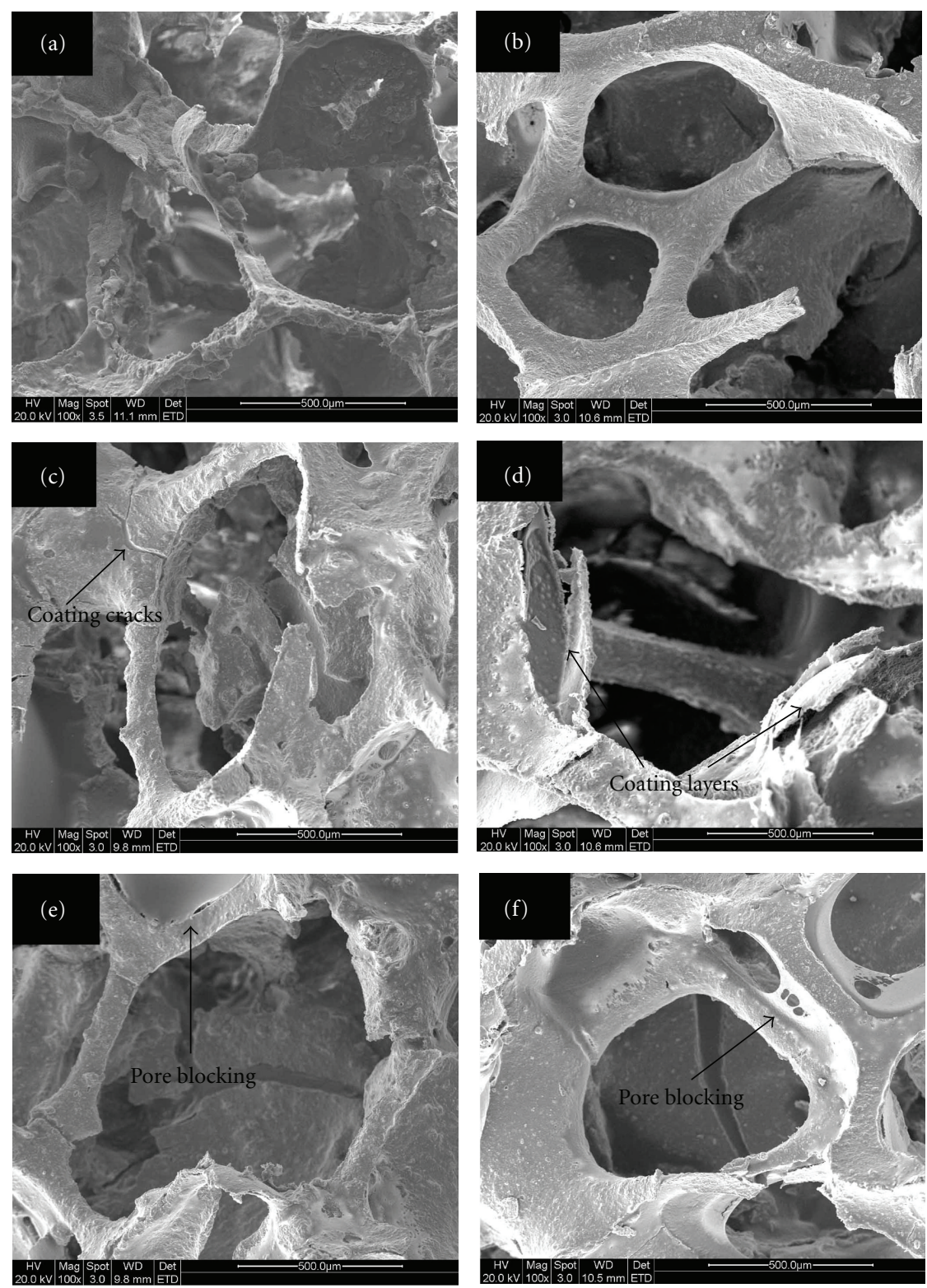

FIGURE 4: ESEM images of the morphologies and microstructures of the BCP scaffolds coated with different layers of HA/PLLA nanocomposites: (a) without coating, (b) monolayer coating, (c) two-layer coating, (d) three-layer coating, (e) four-layer coating, and (f) five-layer coating.

the coating layers were observed clearly while the $\mathrm{BCP}$ scaffold coated with three layers of nanocomposites, which was most likely attributed to low-hydrophilicity property of HA/PLLA nanocomposites (Figure 4(d)). For BCP scaffolds coated with four layers and five layers of nanocomposites, the interconnected porous structure of scaffolds was influenced by the pore blocking slightly (Figures $4(\mathrm{e})$ and $4(\mathrm{f})$ ).

\subsection{Scaffold Porosity and Compressive Strengths' Analysis.} Figure 5 showed the porosity of the BCP scaffolds coated with different layers of HA/PLLA nanocomposites. The porosity of the scaffold without coating was about $96.8 \%$, and it decreased while the scaffold coated with HA/PLLA nanocomposites. As the layers of HA/PLLA nanocomposites increased from 1 to 5 , the porosity of the scaffolds gradually decreased from $94.9 \%$ to $84.4 \%$; however, the compressive strength increased from 2.09 to $10.93 \mathrm{MPa}$ (Figure 6). The compressive strength of $\mathrm{BCP}$ scaffold without coating was about $0.31 \mathrm{MPa}$, which was lower than all BCP scaffolds coated with different layers of HA/PLLA nanocomposites. The BCP scaffold without coating presented the ceramic property, and the struts of scaffolds were reinforced by the coating of HA/PLLA nanocomposites. It could be deduced that the coating layer number has strong influence on the porosity and compressive strength of BCP scaffolds. The results illustrated that $\mathrm{BCP}$ scaffold coated with threelayer HA/PLLA nanocomposites was appropriate in the BCP matrix when comprehensively considering the requirement 


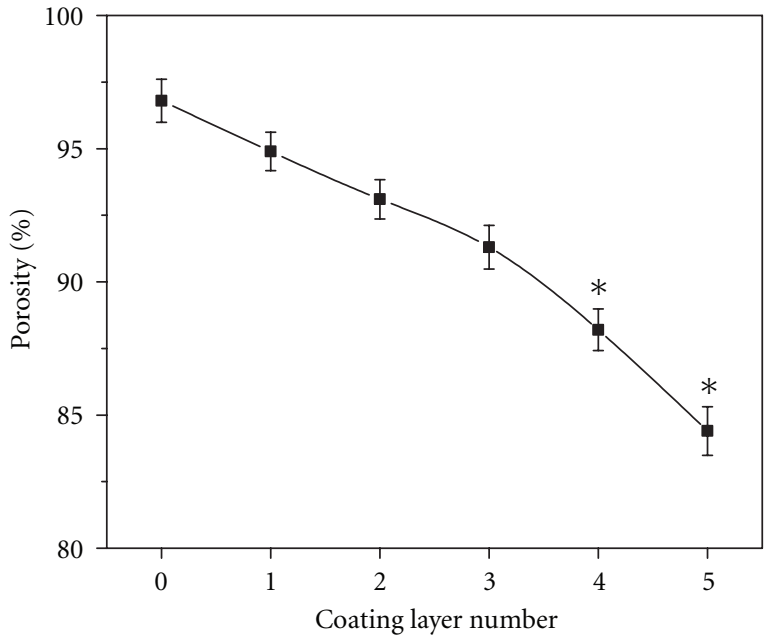

FIGURE 5: The porosity of the BCP scaffolds coated with different layers of HA/PLLA nanocomposites. Error bars represent the mean \pm standard deviation, $n=5, P<0.05$.

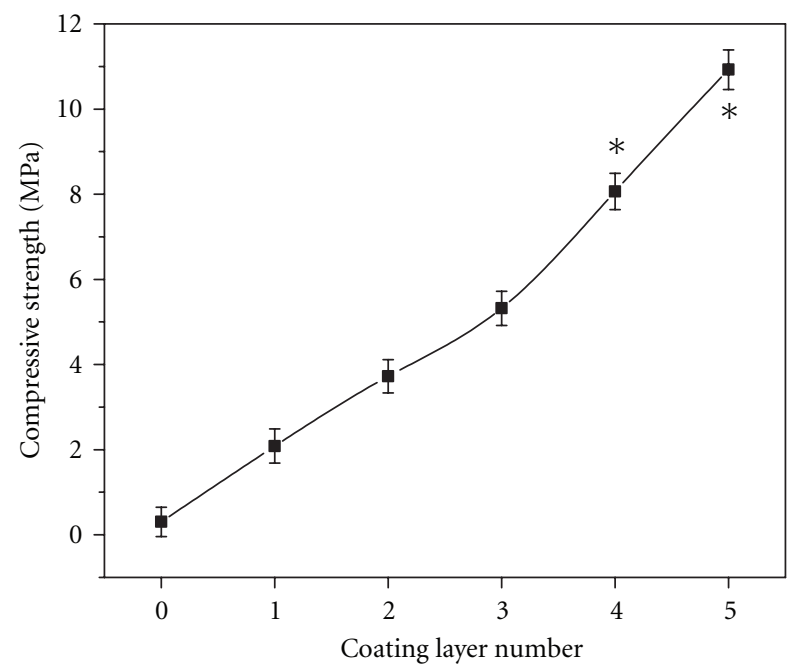

Figure 6: The compressive strengths of the BCP scaffolds coated with different layers of HA/PLLA nanocomposites. Error bars represent the mean \pm standard deviation, $n=5, P<0.05$.

of porosity and mechanical strength for tissue engineering scaffold.

3.5. Biodegradation in Simulated Body Fluid. Figure 7 represents the weight loss of the scaffolds soaked in SBF solution after different soaking times. After 28 days of soaking, the weight loss of BCP scaffold without coating was about $26 \%$ relative to initial weight and showed a greater weight loss than those scaffolds with coating layers of HA/PLLA nanocomposites. The weight loss of BCP scaffolds coated with multilayer of HA/PLLA nanocomposites were decreased at different soaking times compared to BCP scaffold without coating. And the weight loss decreased with the increase

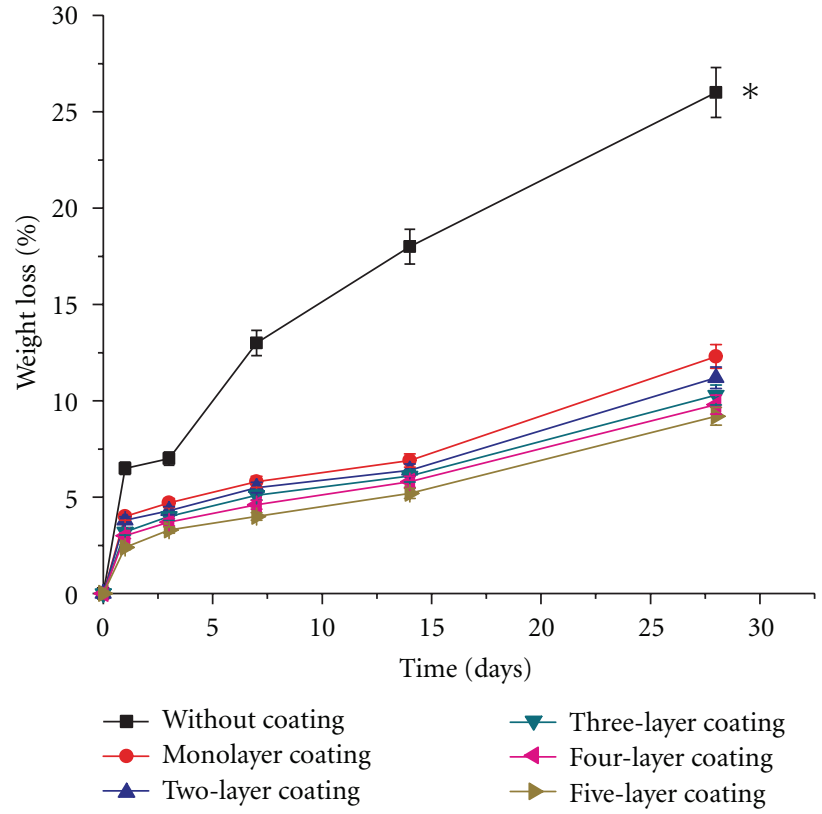

Figure 7: The weight loss of the BCP scaffolds coated with multilayer of HA/PLLA nanocomposites in SBF solution after different soaking times. The weight loss of scaffolds decreased with the increase of coating layer number. Error bars represent the mean \pm standard deviation, $n=5, P<0.05$.

of coating number for prepared scaffolds. The dissolution of BCP nanoparticles in SBF was prevented by the coating of HA/PLLA nanocomposites. The result indicated that the degradation of BCP scaffold could be controlled by the coating layers of HA/PLLA nanocomposites. The graphs of the $\mathrm{pH}$ changes in terms of soaking time also illustrate the dissolution behavior of prepared scaffolds as shown in Figure 8 . The $\mathrm{pH}$ values of SBF without scaffolds were stable throughout the experimental period. The $\mathrm{pH}$ values of $\mathrm{BCP}$ scaffold without coating in SBF solution exhibited approximately 7.40-7.61, and the lower variation was observed. For BCP scaffolds coated with HA/PLLA nanocomposites, the $\mathrm{pH}$ values in SBF solution changed from 6.87 to 7.40 , and the lowest $\mathrm{pH}$ values in SBF solution decreased with the increase of coating number of HA/PLLA nanocomposites.

3.6. In Vitro Cytotoxicity Analysis. MTT test was usually used for evaluating the cell toxicity of biomaterials. In this study, rat osteoblast cells were cultured on six groups of porous scaffolds for 1,3 , and 5 days; respectively, after treatment of the cell-scaffold constructs with the MTT solution, dark blue crystals of formazan were seen, indicating the presence of metabolically active cells. The MTT absorption was measured at $570 \mathrm{~nm}$ with a background subtraction at $630 \mathrm{~nm}$, a higher absorbance indicates that there are either more cells or that they are metabolizing [38], and the results were presented in Figure 9. The reason for which might be owing to the surface morphologies and excellent bioactivity of BCP [39-41]. The absorbance of BCP scaffolds coated with HA/PLLA nanocomposites was higher compared to the negative group at different days, and the BCP scaffold 


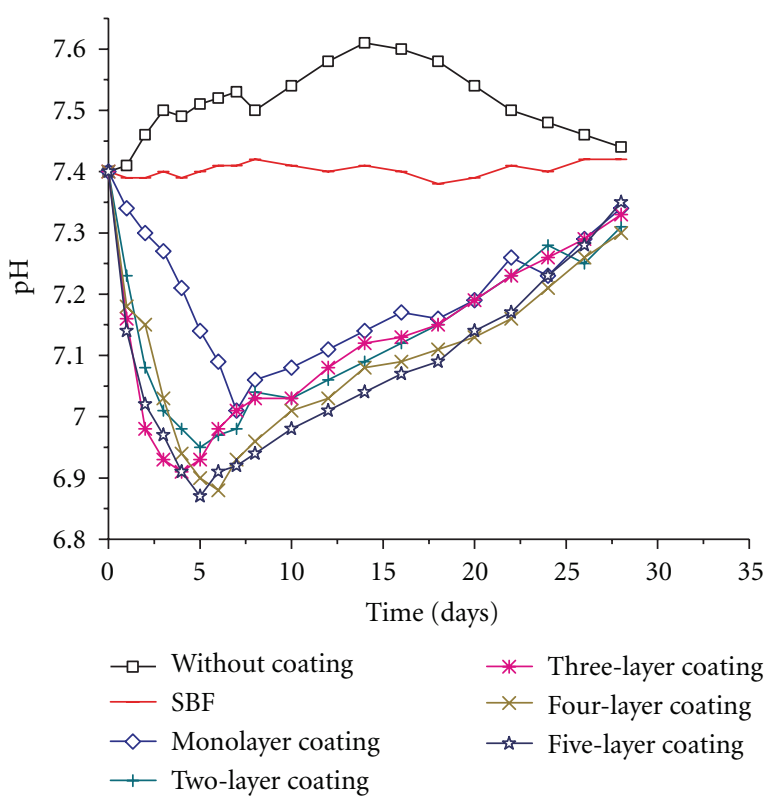

FIGURE 8: The pH value of SBF solution after different soaking times by the BCP scaffolds coated with different layers of HA/PLLA nanocomposites.

coated with three-layer coating exhibited highest absorbance. Therefore, the biphasic calcium phosphate scaffolds coated with multilayer of HA/PLLA nanocomposites prepared in this study can be a promising biomaterial for bone tissue regeneration application due to their good biocompatibility.

3.7. In Vivo Experiment Analysis. The BCP scaffolds coated with three layers of HA/PLLA nanocomposites implanted in the thigh muscle of the SD rat. After operation, the implant site did not show any inflammation such as red swelling and effusion in the skin. No adverse reaction of the animal was observed. Histological images of the tissue section of the implanted site are shown in Figure 10. After 1 day, inflammatory cells like neutrophil, eosinophil, lymphocyte, plasma cell, and macrophage were found to be close to the implant-tissue interface compared to the control group in Figures 10(a) and 10(f). After 7 days, the appearance and shape of the implanted material showed no obvious change, and the amount of inflammatory cells decreased rapidly. After 14 days, the slight connection between the material and surrounding tissue was observed, and the number of fibroblasts increased with increasing time. The disappearance of the inflammatory cells was clearly seen, and their amount was low. Mature fibroblasts were found to form a hyperplastic fibrous tissue surrounding the materials in Figure 10(c). After 21 days after implantation, the connection between material and tissue occurred, and there were slight changes in the appearance and shape of the material. A dense, thin fibrous tissue layer was built in the interface. The surface of materials became roughness, and the semitransparent connection fibrous tissue layer in the interface was observed. Furthermore, the bone-like apatite layer was built obviously in the interface of scaffold. After 28 days after implantation,
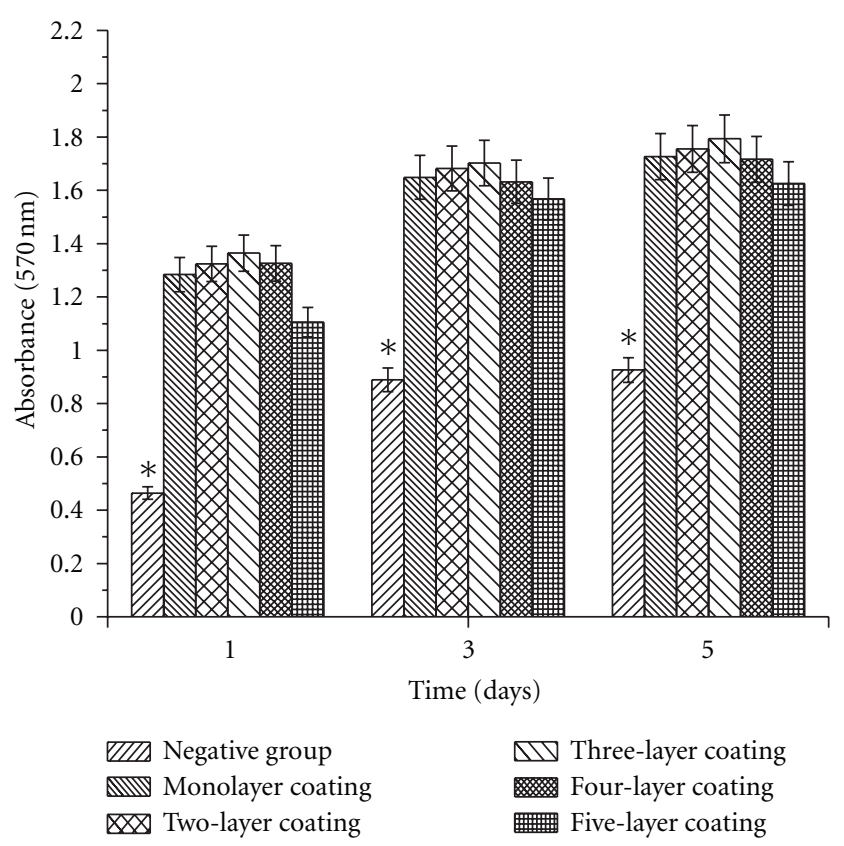

FIGURE 9: Formazan absorption at $570 \mathrm{~nm}$ in MTT assays rat osteoblasts seeded on the BCP scaffolds coated with multilayer of HA/PLLA nanocomposites after 1,3 , and 5 days and the negative control group. Data were expressed as means \pm standard deviation, $n=5, P<0.05$.

the thickness of bone-like layer was enhanced, and the blood cells were found in Figure 10(e), which means the blood vessels grown into the pores of the materials. Implantation experiment in muscle experiment revealed that BCP scaffolds coated with multilayer of HA/PLLA nanocomposites were clearly nontoxic.

\section{Conclusion}

Well-developed BCP scaffolds coated with multilayer of HA/PLLA nanocomposites with interconnectivity, high porosity, and moderate compressive strength as well as good biocompatibility were fabricated for bone tissue engineering. After coated with HA/PLLA nanocomposites, the scaffolds maintained the BCP framework structure, and the porous network structure of scaffolds remained unchanged; however, the compressive strength was increased with the increase of coating layer number of HA/PLLA nanocomposites. The prepared scaffolds showed the lower variation of $\mathrm{pH}$ values in SBF solution, and an increase of coating layer number led to the decrease of the biodegradation rate at different days. Moreover, the multilayer coating scaffolds had good cytocompatibility, showing no negative effects on cells growth and proliferation. Furthermore, the bonelike apatite layer was built obviously in the interface of scaffold after 21 days after implantation in SD rat muscle. In conclusion, the BCP scaffold coated with multilayer of HA/PLLA nanocomposites could be a candidate as an excellent substitute for damaged or defect bone in bone tissue engineering. Further animal bone defect experiments will be 


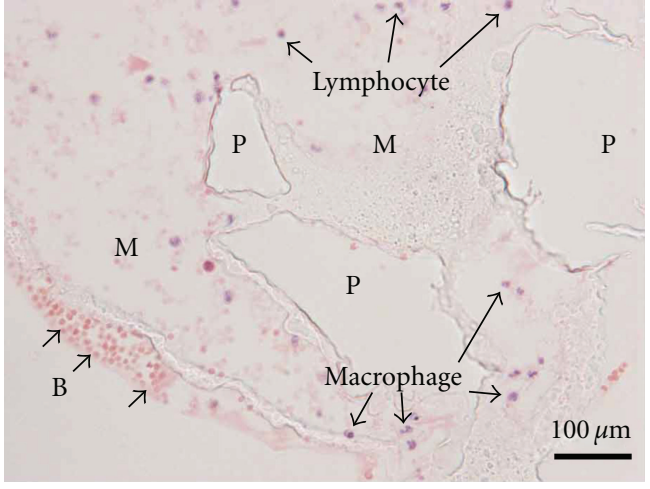

(a) 1 day

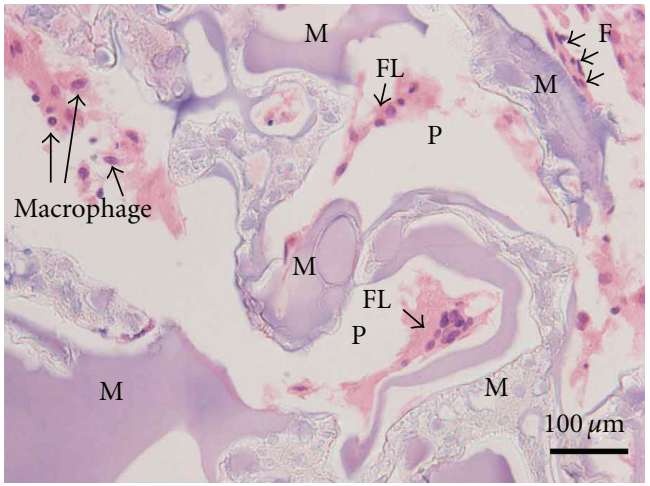

(c) 14 days

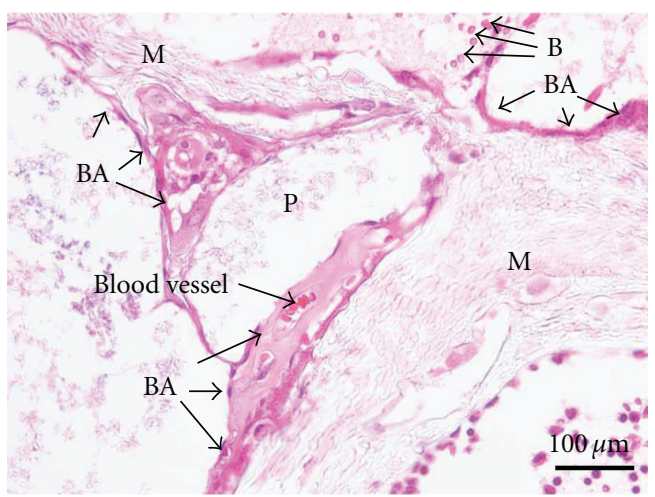

(e) 28 days

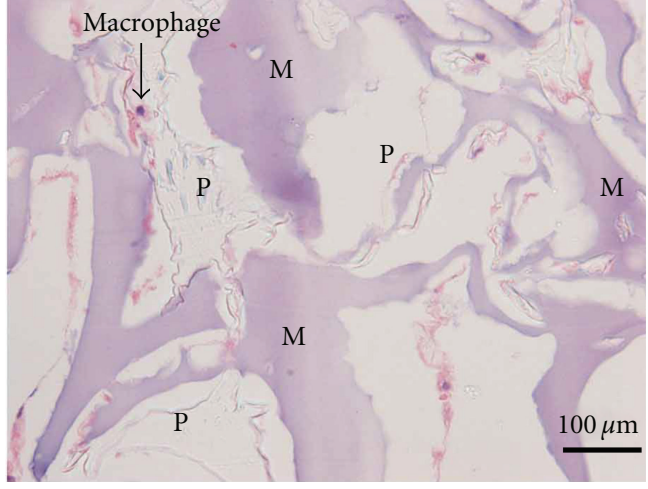

(b) 7 days

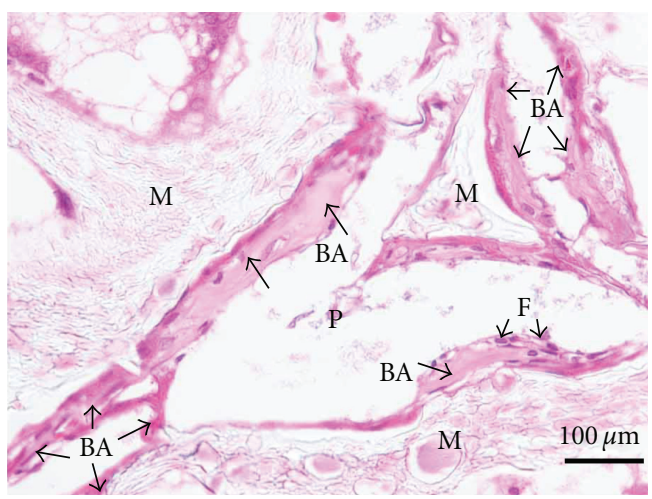

(d) 21 days

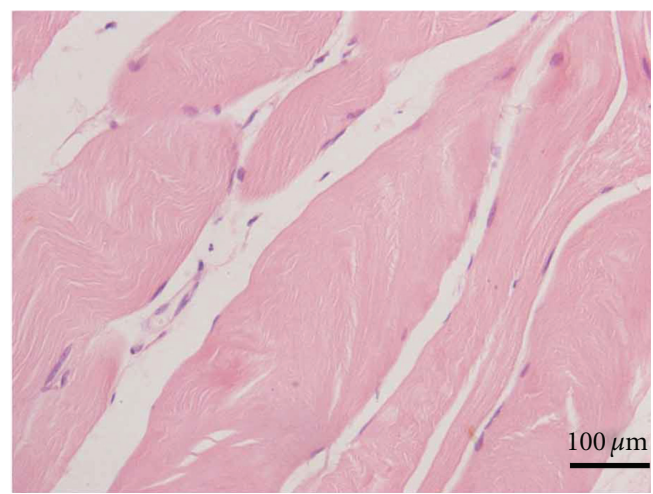

(f) Control

Figure 10: Optical micrographs of HE staining slices of the BCP scaffolds coated with three layers of HA/PLLA nanocomposites in muscle of SD rats at indicated days. The normal muscle tissue was taken as the blank control ( $\mathrm{M}$ means materials; P means the pores; $\mathrm{B}$ means blood cells; F means fibroblasts; FL means fibrous tissue layer; BA means bone-like apatite. Scale bar: $100 \mu \mathrm{m}$ ).

carried out to demonstrate its capacity for bone repair and regeneration.

\section{Acknowledgments}

The authors acknowledge the Independent Innovation Foundation of Huazhong University of Science and Technology (2010JC029/2011JC042). Technical assistance from the Analytical and Testing Center of HUST is gratefully acknowledged.

\section{References}

[1] M. Doblaré, J. M. García, and M. J. Gómez, "Modelling bone tissue fracture and healing: a review," Engineering Fracture Mechanics, vol. 71, no. 13-14, pp. 1809-1840, 2004.

[2] V. M. Goldberg and S. Stevenson, "Bone graft options: fact and fancy," Orthopedics, vol. 17, no. 9, pp. 809-821, 1994.

[3] V. Karageorgiou and D. Kaplan, "Porosity of 3D biomaterial scaffolds and osteogenesis," Biomaterials, vol. 26, no. 27, pp. 5474-5491, 2005. 
[4] S. J. Hollister, "Scaffold design and manufacturing: from concept to clinic," Advanced Materials, vol. 21, no. 32-33, pp. 3330-3342, 2009.

[5] K. J. L. Burg, S. Porter, and J. F. Kellam, "Biomaterial developments for bone tissue engineering," Biomaterials, vol. 21, no. 23, pp. 2347-2359, 2000.

[6] D. W. Hutmacher, "Scaffolds in tissue engineering bone and cartilage," Biomaterials, vol. 21, no. 24, pp. 2529-2543, 2000.

[7] S. V. Dorozhkin, "Bioceramics of calcium orthophosphates," Biomaterials, vol. 31, no. 7, pp. 1465-1485, 2010.

[8] J. M. Curran, J. A. Gallagher, and J. A. Hunt, "The inflammatory potential of biphasic calcium phosphate granules in osteoblast/macrophage co-culture," Biomaterials, vol. 26, no. 26, pp. 5313-5320, 2005.

[9] R. Z. LeGeros, S. Lin, R. Rohanizadeh, D. Mijares, and J. P. LeGeros, "Biphasic calcium phosphate bioceramics: preparation, properties and applications," Journal of Materials Science, vol. 14, no. 3, pp. 201-209, 2003.

[10] S. Kano, A. Yamazaki, R. Otsuka, M. Ohgaki, M. Akao, and H. Aoki, "Application of hydroxyapatite-sol as drug carrier," Biomedical Materials and Engineering, vol. 4, no. 4, pp. 283290, 1994.

[11] C. Stähli, M. Bohner, M. Bashoor-Zadeh, N. Doebelin, and G. Baroud, "Aqueous impregnation of porous $\beta$-tricalcium phosphate scaffolds," Acta Biomaterialia, vol. 6, no. 7, pp. 2760 2772, 2010.

[12] H. Yuan, C. A. Van Blitterswijk, K. De Groot, and J. D. De Bruijn, "Cross-species comparison of ectopic bone formation in biphasic calcium phosphate (BCP) and hydroxyapatite (HA) scaffolds," Tissue Engineering, vol. 12, no. 6, pp. 16071615, 2006.

[13] K. Rezwan, Q. Z. Chen, J. J. Blaker, and A. R. Boccaccini, "Biodegradable and bioactive porous polymer/inorganic composite scaffolds for bone tissue engineering," Biomaterials, vol. 27, no. 18, pp. 3413-3431, 2006.

[14] H. W. Kim, J. C. Knowles, and H. E. Kim, "Hydroxyapatite/ poly( $\varepsilon$-caprolactone) composite coatings on hydroxyapatite porous bone scaffold for drug delivery," Biomaterials, vol. 25, no. 7-8, pp. 1279-1287, 2004.

[15] X. Miao, L. P. Tan, L. S. Tan, and X. Huang, "Porous calcium phosphate ceramics modified with PLGA-bioactive glass," Materials Science and Engineering C, vol. 27, no. 2, pp. 274279, 2007.

[16] I. K. Jun, J. H. Song, W. Y. Choi, Y. H. Koh, H. E. Kim, and H. W. Kim, "Porous hydroxyapatite scaffolds coated with bioactive apatite-wollastonite glass-ceramics," Journal of the American Ceramic Society, vol. 90, no. 9, pp. 2703-2708, 2007.

[17] T. Tian, D. Jiang, J. Zhang, and Q. Lin, "Fabrication of bioactive composite by developing PLLA onto the framework of sintered HA scaffold," Materials Science and Engineering C, vol. 28, no. 1, pp. 51-56, 2008.

[18] X. Miao, D. M. Tan, J. Li, Y. Xiao, and R. Crawford, "Mechanical and biological properties of hydroxyapatite/tricalcium phosphate scaffolds coated with poly(lactic-co-glycolic acid)," Acta Biomaterialia, vol. 4, no. 3, pp. 638-645, 2008.

[19] J. Zhao, L. Y. Guo, X. B. Yang, and J. Weng, "Preparation of bioactive porous HA/PCL composite scaffolds," Applied Surface Science, vol. 255, no. 5, pp. 2942-2946, 2008.

[20] C. Wu, Y. Ramaswamy, P. Boughton, and H. Zreiqat, "Improvement of mechanical and biological properties of porous $\mathrm{CaSiO}_{3}$ scaffolds by poly(d,l-lactic acid) modification," Acta Biomaterialia, vol. 4, no. 2, pp. 343-353, 2008.
[21] J. Zhao, X. Lu, K. Duan, L. Y. Guo, S. B. Zhou, and J. Weng, "Improving mechanical and biological properties of macroporous HA scaffolds through composite coatings," Colloids and Surfaces B, vol. 74, no. 1, pp. 159-166, 2009.

[22] S. I. R. Esfahani, F. Tavangarian, and R. Emadi, "Nanostructured bioactive glass coating on porous hydroxyapatite scaffold for strength enhancement," Materials Letters, vol. 62, no. 19, pp. 3428-3430, 2008.

[23] A. Bianco, I. Cacciotti, M. Lombardi, L. Montanaro, and G. Gusmano, "Thermal stability and sintering behaviour of hydroxyapatite nanopowders," Journal of Thermal Analysis and Calorimetry, vol. 88, no. 1, pp. 237-243, 2007.

[24] T. Koshino, T. Murase, T. Takagi, and T. Saito, "New bone formation around porous hydroxyapatite wedge implanted in opening wedge high tibial osteotomy in patients with osteoarthritis," Biomaterials, vol. 22, no. 12, pp. 1579-1582, 2001.

[25] A. E. Porter, N. Patel, J. N. Skepper, S. M. Best, and W. Bonfield, "Effect of sintered silicate-substituted hydroxyapatite on remodelling processes at the bone-implant interface," Biomaterials, vol. 25, no. 16, pp. 3303-3314, 2004.

[26] S. Gay, S. Arostegui, and J. Lemaitre, "Preparation and characterization of dense nanohydroxyapatite/PLLA composites," Materials Science and Engineering C, vol. 29, no. 1, pp. 172$177,2009$.

[27] S. K. Lan Levengood, M. J. Poellmann, S. G. Clark, D. A. Ingram, M. C. Yoder, and A. J. Wagoner Johnson, "Human endothelial colony forming cells undergo vasculogenesis within biphasic calcium phosphate bone tissue engineering constructs," Acta Biomaterialia, vol. 7, pp. 4222-4228, 2011.

[28] S. Schaefer, R. Detsch, F. Uhl, U. Deisinger, and G. Ziegler, "How degradation of calcium phosphate bone substitute materials is influenced by phase composition and porosity," Advanced Engineering Materials, vol. 13, no. 4, pp. 342-350, 2011.

[29] R. P. Félix Lanao, S. C. G. Leeuwenburgh, J. G. C. Wolke, and J. A. Jansen, "In vitro degradation rate of apatitic calcium phosphate cement with incorporated PLGA microspheres," Acta Biomaterialia, vol. 7, no. 9, pp. 3459-3468, 2011.

[30] G. Sui, X. Yang, F. Mei et al., "Poly-L-lactic acid/hydroxyapatite hybrid membrane for bone tissue regeneration," Journal of Biomedical Materials Research, vol. 82, no. 2, pp. 445-454, 2007.

[31] S. I. Jeong, E. K. Ko, J. Yum, C. H. Jung, Y. M. Lee, and H. Shin, "Nanofibrous poly(lactic acid)/hydroxyapatite composite scaffolds for guided tissue regeneration," Macromolecular Bioscience, vol. 8, no. 4, pp. 328-338, 2008.

[32] S. I. Roohani-Esfahani, S. Nouri-Khorasani, Z. Lu, R. Appleyard, and H. Zreiqat, "The influence hydroxyapatite nanoparticle shape and size on the properties of biphasic calcium phosphate scaffolds coated with hydroxyapatite-PCL composites," Biomaterials, vol. 31, no. 21, pp. 5498-5509, 2010.

[33] S. I. Roohani-Esfahani, S. Nouri-Khorasani, Z. F. Lu, R. C. Appleyard, and H. Zreiqat, "Effects of bioactive glass nanoparticles on the mechanical and biological behavior of composite coated scaffolds," Acta Biomaterialia, vol. 7, no. 3, pp. 1307$1318,2011$.

[34] H. H. Jin, S. H. Min, Y. K. Song, H. C. Park, and S. Y. Yoon, "Degradation behavior of poly(lactide-co-glycolide) $/ \beta$-TCP composites prepared using microwave energy," Polymer Degradation and Stability, vol. 95, no. 9, pp. 1856-1861, 2010.

[35] S. M. Choi and H. Awaji, "Nanocomposites-a new material design concept," Science and Technology of Advanced Materials, vol. 6 , no. 1, pp. 2-10, 2005. 
[36] H. Awaji, S. M. Choi, and E. Yagi, "Mechanisms of toughening and strengthening in ceramic-based nanocomposites," Mechanics of Materials, vol. 34, no. 7, pp. 411-422, 2002.

[37] C. C. Chen, J. Y. Chueh, H. Tseng, H. M. Huang, and S. Y. Lee, "Preparation and characterization of biodegradable PLA polymeric blends," Biomaterials, vol. 24, no. 7, pp. 1167-1173, 2003.

[38] M. M. Stevens, H. F. Qanadilo, R. Langer, and V. P. Shastri, "A rapid-curing alginate gel system: utility in periosteum-derived cartilage tissue engineering," Biomaterials, vol. 25, no. 5, pp. 887-894, 2004.

[39] H. W. Kim, H. E. Kim, and V. Salih, "Stimulation of osteoblast responses to biomimetic nanocomposites of gelatin-hydroxyapatite for tissue engineering scaffolds," Biomaterials, vol. 26, no. 25, pp. 5221-5230, 2005.

[40] H. W. Kim, J. H. Song, and H. E. Kim, "Nanofiber generation of gelatin-hydroxyapatite biomimetics for guided tissue regeneration," Advanced Functional Materials, vol. 15, no. 12, pp. 1988-1994, 2005.

[41] P. L. Lin, H. W. Fang, T. Tseng, and W. H. Lee, "Effects of hydroxyapatite dosage on mechanical and biological behaviors of polylactic acid composite materials," Materials Letters, vol. 61, no. 14-15, pp. 3009-3013, 2007. 

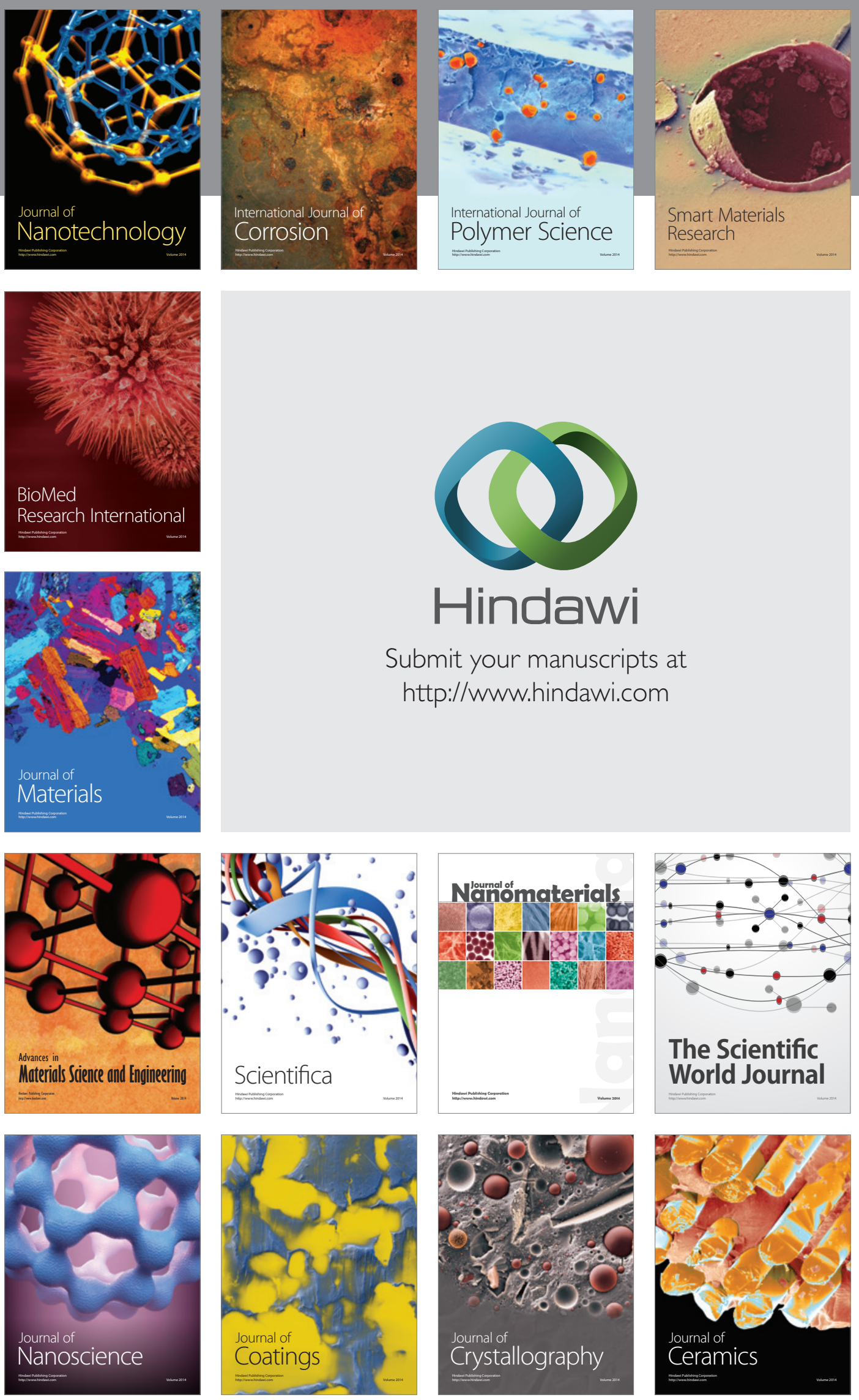

The Scientific World Journal

Submit your manuscripts at

http://www.hindawi.com

\section{World Journal}

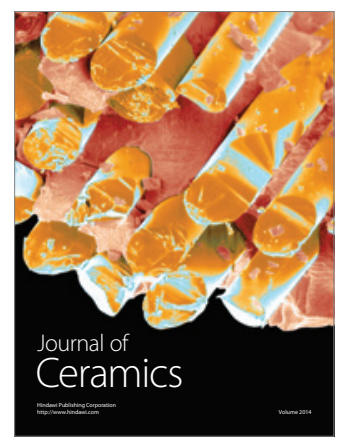

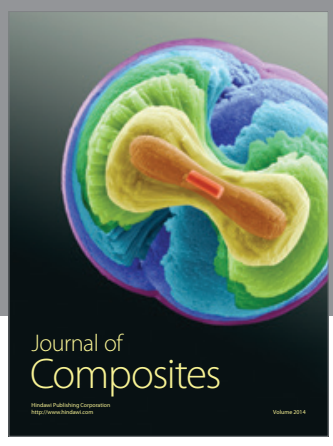
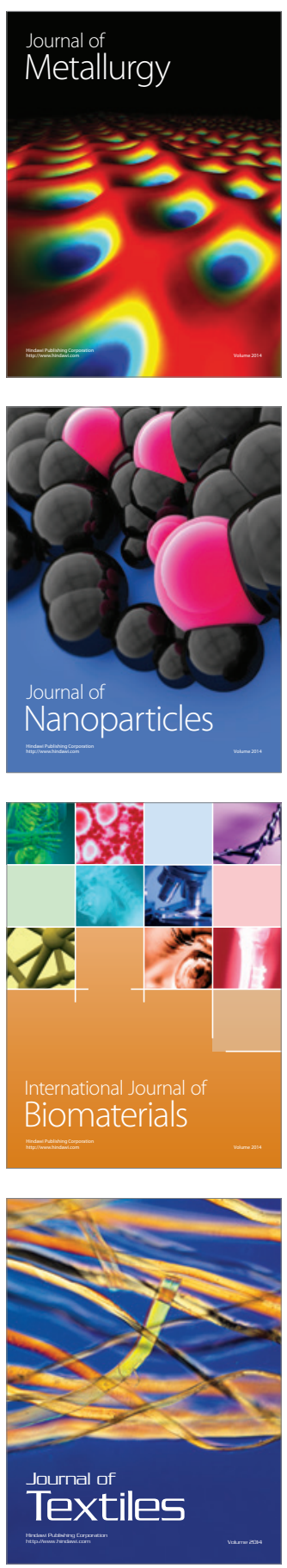